\title{
A Novel Antibody Targeting Tau Phosphorylated at Serine 235 Detects Neurofibrillary Tangles
}

\author{
David Brici, Jürgen Götz and Rebecca M. Nisbet* \\ Clem Jones Centre for Ageing Dementia Research, Queensland Brain Institute, The University of Queensland, \\ St Lucia Campus, Brisbane, QLD, Australia
}

Handling Associate Editor: Rakez Kayed

Accepted 6 October 2017

\begin{abstract}
Alzheimer's disease is characterized by two main pathological hallmarks in the human brain: the extracellular deposition of amyloid- $\beta$ as plaques and the intracellular accumulation of the hyperphosphorylated protein tau as neurofibrillary tangles (NFTs). Phosphorylated tau (p-tau) specific-antibodies and silver staining have been used to reveal three morphological stages of NFT formation: pre-NFTs, intraneuronal NFTs (iNFTs), and extraneuronal NFTs (eNFTs). Here we characterize a novel monoclonal antibody, RN235, which is specific for tau phosphorylated at serine 235, and detects iNFTs and eNFTs in brain tissue, suggesting that phosphorylation at this site is indicative of late stage changes in tau.
\end{abstract}

Keywords: Alzheimer's disease, antibody, frontotemporal lobar degeneration, neurofibrillary tangle, tau

\section{INTRODUCTION}

Alzheimer's disease (AD) is characterized pathologically by the extracellular accumulation of amyloid plaques, the intracellular accumulation of neurofibrillary tangles (NFTs), and extensive neuronal cell death. NFTs are also the primary pathology observed in related tauopathies, including frontotemporal lobar degeneration-tau (FTLD-tau), corticobasal degeneration, and progressive supranuclear palsy. The major constituent of NFTs is the microtubule-associated protein tau which has undergone hyperphosphorylation and self-aggregation to

\footnotetext{
${ }^{*}$ Correspondence to: Rebecca Nisbet, Clem Jones Centre for Ageing Dementia Research (CJCADR), Queensland Brain Institute, The University of Queensland, St Lucia (Brisbane), QLD 4072, Australia. Tel.: +61 73346 66326; Fax: +61 7334 66301; E-mail: r.nisbet@uq.edu.au.
}

form insoluble fibers known as straight and paired helical filaments.

Using phosphorylated tau (p-tau)-specific antibodies and silver staining, three morphological stages of NFT formation have been distinguished: 1) pre-NFTs, 2) intraneuronal NFTs (iNFTs), and 3) extraneuronal NFTs (eNFTs) [1]. Pre-NFTs are defined by diffuse, sometimes punctate, tau staining within the cytoplasm of otherwise normal-looking neurons, with well-preserved dendrites and a central nucleus; they are reactive with anti-tau antibodies but not silver stain [2]. iNFTs contain aggregated filamentous structures and are found within the cytoplasm of neurons which have a definable but frequently displaced nucleus. Typically, the dendrites of iNFT-affected neurons appear to have deteriorated but retain their position. Finally, eNFTs result from the death of the tangle-bearing neurons, and 
are identifiable by the absence of neuronal nuclei and stainable cytoplasm [2]. Previous studies have shown that antibodies specific for tau phosphorylated at Thr153, Ser262, and Thr231 and certain conformational epitopes recognized by the antibodies MC1 and ALZ50 label pre-NFTs, whereas AT8 (Ser202/Thr205) and PHF1 (Ser396/Ser404) antibodies preferentially identify iNFTs and eNFTs [1].

A previously uncharacterized tau epitope, phosphorylated Ser235 (p-Ser235), which is located in the proline-rich region of tau, has also been shown to be elevated in $\mathrm{AD}$ [3]. Furthermore, in vitro studies have demonstrated that phosphorylation of Ser235 abolishes the ability of tau to polymerize tubulin into microtubules [4], which could be due to disturbances in the local secondary structure of tau upon phosphorylation at this site $[5,6]$. An antibody specific for p-Ser235 may therefore be useful for the characterization of tau pathology. Here, we demonstrate that a novel anti-pSer235 antibody, RN235, specifically detects tau aggregates consistent with the transition from iNFTs to eNFTs, suggesting that phosphorylation of this site is associated with late p-tau changes.

\section{MATERIALS AND METHODS}

\section{Antibody generation}

RN235 is a monoclonal antibody raised against the p-tau peptide VAVVRT(p)PPKS(p)PS (phosphorylated residues Thr231 and Ser235 of the longest human tau isoform, tau441) (Beijing Protein Innovation). The hybridoma was cultured at $37^{\circ} \mathrm{C}$ with $5 \%$ $\mathrm{CO}_{2}$ in CD Hybridoma medium (Life Technologies) supplemented with $8 \mathrm{mM}$ Glutamax (Life Technologies). IgG was purified by affinity chromatography using a protein A column, then buffer exchanged into $1 \mathrm{x}$ phosphate-buffered saline (PBS) (Protein Expression Facility, The University of Queensland). The isotype of RN235 was characterized using the IsoStrip Mouse Monoclonal Antibody Isotyping kit (Sigma-Aldrich) and determined to be IgG1 with a kappa light chain. Biotinylation of RN235 was conducted using the EZ-link ${ }^{\mathrm{TM}}$ Micro NHS-PEG4Biotinylation kit (Thermo Fisher Scientific).

\section{Recombinant human tau}

cDNA encoding full-length human tau was cloned into the pET-DEST42 vector (Thermo Fisher Scientific) in frame with the C-terminal His6 and V5 tags. Plasmids were transformed into One Shot BL21 bacterial cells (Thermo Fisher Scientific) and recombinant protein expression was induced with $1 \mathrm{mM}$ IPTG. Protein purification was conducted following the protocol outlined in [7].

\section{In vitro GSK-3 phosphorylation}

In vitro phosphorylation of recombinant human tau was conducted as previously described [8]. Briefly, $10 \mu \mathrm{M}$ tau was incubated with 9 units of GSK$3 \beta$ and $80 \mu \mathrm{M}$ ATP in kinase buffer (New England Biosciences) at $30^{\circ} \mathrm{C}$ for $8 \mathrm{~h}$.

\section{Dot blot}

Phosphorylated and non-phosphorylated human tau were dot-blotted onto a nitrocellulose membrane and detected with RN235 or Tau5 (Merck).

\section{ELISA}

The binding specificity and EC50 of RN235 was determined using an enzyme-linked immunosorbent assay (ELISA) as previously described [8]. For binding specificity, Immuno 96 MicroWell plates (Nunc) were coated with $10 \mu \mathrm{g} / \mathrm{ml}$ recombinant full-length human tau (+/- phosphorylation), followed by incubation with RN235 or control IgG at $10 \mu \mathrm{g} / \mathrm{ml}$. For EC50 calculation, plates were coated with $10 \mu \mathrm{g} / \mathrm{ml}$ bovine serum albumin (BSA)-conjugated phosphopeptides in PBS, followed by incubation with serial dilutions of RN235 in PBS. For all assays, plates were blocked in $3 \%$ BSA and bound antibodies were detected with anti-mouse horseradish peroxidase (HRP) conjugate $(1: 5,000)$, followed by incubation with the substrate 3,3',5,5'-tetramethylbenzidine (Sigma-Aldrich). The absorbance was measured at $450 \mathrm{~nm}(\mathrm{BMG})$ and measurements were the result of three readings after subtraction of the absorbance of PBS alone.

Mice

All animal experiments were conducted under the guidelines of the Australian Code of Practice for the Care and Use of Animals for Scientific Purposes and approved by the University of Queensland Animal Ethics Committee (QBI/412/14/NHMRC; QBI/027/12/NHMRC). K3 mice express human 1N4R tau with the K396I mutation under the control of the murine Thy 1.2 promoter [9]. $\mathrm{rTg} 4510$ mice express human 0N4R tau with the P301L mutation, 
ALZ17 mice express wild-type human 2N4R tau and pR5 mice express human 2N4R tau with the P301L mutation. At the specified ages, the mice were anaesthetized and transcardially perfused with PBS. Their brains were then harvested and immersion-fixed in 4\% paraformaldehyde (PFA) (Sigma-Aldrich) for immunohistochemical analysis.

\section{Human brain tissue}

Sections of postmortem human brain tissue from donors diagnosed with AD or FTLD-tau and controls were obtained from the Brain Bank for Aging Research (Tokyo Metropolitan Geriatric Hospital \& Institute of Gerontology). For all brain tissue, clinical and pathological information was obtained under prior written consent from the brain donors and their next of kin. All work was approved by the University of Queensland Institutional Human Research Ethics Committee (approval number 2012000951).

\section{Histology}

PFA-fixed brains were embedded in paraffin using a benchtop tissue processor (Leica). Immunohistochemistry was performed as previously described [10]. Antigen retrieval was conducted in a domestic microwave (850W) in citrate buffer $\mathrm{pH} 5.8$ for $15 \mathrm{~min}$, followed by cooling at room temperature for $40 \mathrm{~min}$. The primary antibodies used were RN235 $(1: 5,000)$, AT180 $(1: 1,000)$, and AT8 $(1: 1,000)$ (Thermo Fisher Scientific). For DAB staining, a biotin-coupled anti-mouse secondary antibody was used, followed by detection with the ABC-HRP detection kit (Vector) and metal-enhanced DAB (Dako). Counterstaining was achieved with haematoxylin (Dako). For fluorescence labeling, AlexaFluor-conjugated antimouse secondary antibodies were used together with the nuclear stain, DAPI (Sigma). For detection of biotinylated RN235, AlexaFluor-conjugated streptavidin was used. Bielschowsky-silver impregnation of paraffin sections was done as previously described [9]. Imaging was performed using a Metafer Vslide Scanner (MetaSystems) and an Axio Imager Z2 (Zeiss).

\section{Statistical analysis}

Statistical analysis was performed with GraphPad Prism 7.0a software using a two-way ANOVA or student's $t$-test. All values are given as mean \pm SEM.

\section{RESULTS}

RN235 is specific for tau phosphorylated at serine 235

It has been reported that phosphorylation of tau at Thr231 and Ser235 is increased in patients with AD [3]. We generated a murine monoclonal antibody against a peptide with the dual phosphorylation. Dot blot analysis revealed that the antibody was specific to p-tau, with no detectable reactivity to the non-phosphorylated protein (Fig. 1A). This was further confirmed by ELISA (Fig. 1B). The calculated EC50 of RN235 to the dual phosphorylated peptide was $0.41 \mathrm{nM}$ (Fig. 1C). This was consistent with the EC50 calculated against the peptide phosphorylated at Ser 235 alone $(0.63 \mathrm{nM})$, suggesting that RN235 only requires Ser235 phosphorylation and that binding is not disrupted when Thr231 is phosphorylated (Fig. 1C). The EC50 against the p-Thr231 peptide was considerably lower $(407 \mathrm{nM})$, confirming that RN235 is essentially p-Ser235 specific, displaying only a weak interaction with p-Thr231 (Fig. 1C).

\section{RN235 detects compact neuronal aggregates}

To determine if RN235 is capable of detecting p-tau in brain tissue, postmortem cortical samples from human AD and FTLD-tau patients were analyzed (Fig. 2A). RN235 bound to dense tau aggregates without a definable nucleus in the $\mathrm{AD}$ and FTLD-tau tissue, with no binding observed in the control tissue (Fig. 2A). These aggregates exhibited collapsed or displaced dendrites consistent with those of iNFTs and eNFTs (Fig. 2A). Similar aggregates were also observed in the brain tissue from human tau transgenic mice (K3, rTg4510, ALZ17 and pR5), but were absent in tau knockout and wild-type tissue (Fig. 2B). Although present, RN235 labeling in the pR5 mice was very sparse, possibly due to the low expression of human tau and comparatively subtle tau pathology in these mice (Fig. 2B). To better characterize the NFT stage that RN235 detects, co-labeling was conducted in K3 brain tissue with a p-Thr231 antibody, AT180, which preferentially detects pre-NFTs, and with a p-Ser202/205 antibody, AT8, which preferentially detects iNFTs and eNFTs (Fig. 2C). RN235 labeling differed from the heterogeneous labeling observed with AT180, which frequently produced a more diffuse cytoplasmic tau staining within neurons with detectable nuclei, 
A

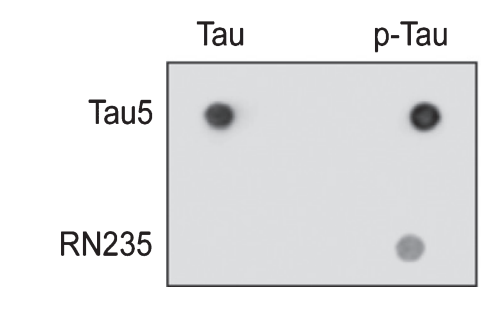

C
B

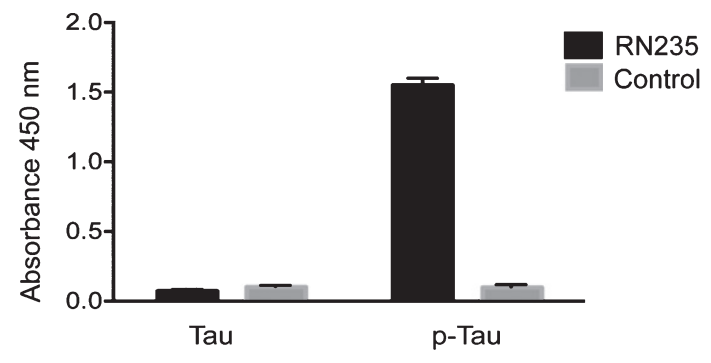

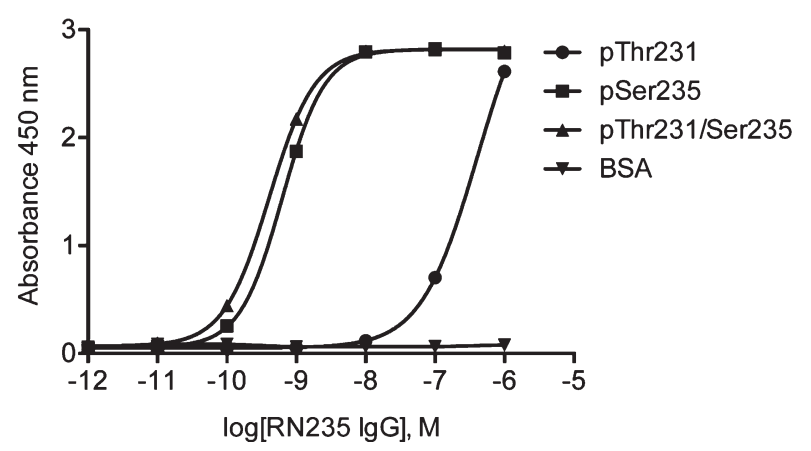

\begin{tabular}{|c|c|}
\hline Peptide & EC50 (M) \\
\hline pThr231 & $4.076 \times 10^{-7}$ \\
\hline pSer235 & $6.272 \times 10^{-10}$ \\
\hline pThr231/Ser235 & $4.064 \times 10^{-10}$ \\
\hline
\end{tabular}

Fig. 1. RN235 monoclonal antibody specifically recognizes tau phosphorylated at Ser235. Dot blot analysis (A) and ELISA (B) reveal that RN235 is specific for phosphorylated tau and has no cross-reactivity with non-phosphorylated tau when compared to a pan-tau antibody, tau5, or a non-specific control antibody. C) The binding activity of RN235 was determined by ELISA using plates coated with dual phosphopeptide (pThr231/Ser235) or individual phospho-peptides (pThr231 or pSer235). The EC50 of the RN235 to pThr231 was calculated to be $407 \mathrm{nM}$, whereas the EC50s of RN235 to the dual phospho-peptide and pSer235 were much higher ( $0.41 \mathrm{nM}$ and $0.63 \mathrm{nM}$, respectively).

consistent with the presence of pre-NFTs, and less frequently co-localized with RN235 (Fig. 2C). On the other hand, AT8 labeling was consistent with that of RN235 and co-localization was frequently observed (Fig. 2C).

\section{RN235 detects a progressive tau pathology}

As phosphorylation of tau at Ser235 is elevated in disease [3], we next wanted to confirm that the tau aggregates detected by RN235 represented a progressive pathology. K3 mice typically exhibit progressive NFT formation from 2 months of age [9]. This was confirmed with Bielschowsky's silver staining which demonstrated an approximate 2-fold increase in NFTs in the cortex of K3 mice between 2 and 14 months (Fig. 3A). In a similar pattern, RN235 labeling revealed that this epitope was approximate 2fold more abundant in the older mice (Fig. 3B), with the RN235 labeling co-localizing with that of AT8 (Fig. 3B). This demonstrates that RN235, similar to AT8, detects a pathological epitope which increases with disease progression.

\section{DISCUSSION}

The progression of hyperphosphorylated tau aggregation from pre-fibrillar aggregates to NFTs can be characterized histologically using p-tau antibodies and silver staining. Here we have demonstrated in both human post-mortem tissue and transgenic mice, that a novel monoclonal antibody, RN235, which is specific for tau phosphorylated at Ser235, detects compact tau aggregates without a definable nucleus and with displaced to collapsed dendrites, consistent with intraneuronal and extraneuronal NFTs. Labeling was dominant in more advanced stages of disease and was rarely observed in mice at young ages or those with only a subtle tau pathology.

Ser235 is commonly phosphorylated in conjunction with Thr231 [11] and both undergo phosphorylation by the kinases GSK-3 $\beta$, cyclindependent kinase 5, and cyclic-AMP-dependent protein kinase. As the activity of GSK-3 $\beta$ is enhanced by tau priming, whereby an amino acid in the $n+4$ site has already been phosphorylated, it has previously been suggested that phosphorylation of Ser235 

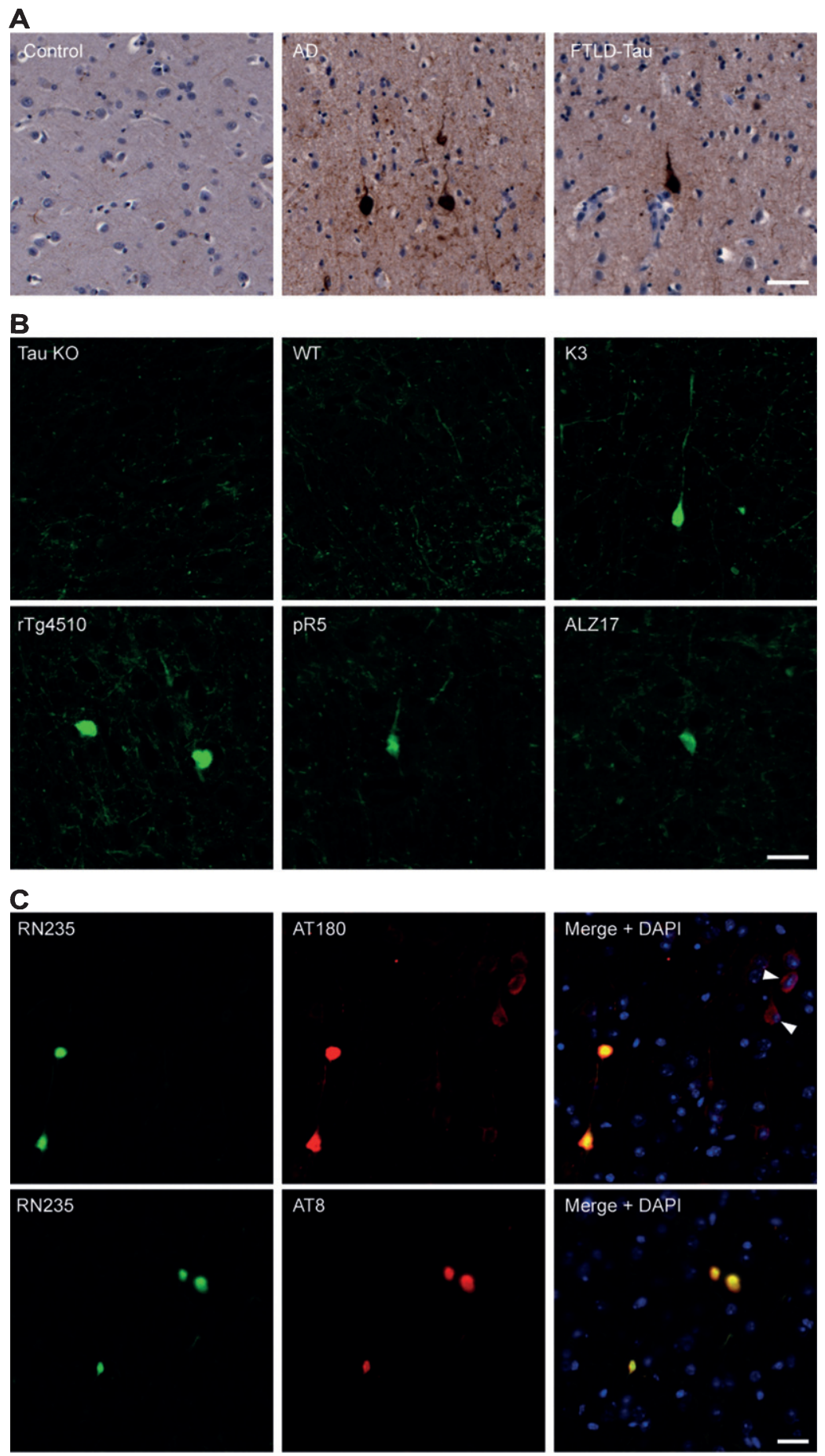

Fig. 2. RN235 detects tau NFTs in human and mouse tissue. A) RN235 detects NFTs which lack a definable nucleus in human cortical tissue from patients with Alzheimer's disease (AD; $n=2$; mean age $=84.5$ years) and frontotemporal lobar degeneration-tau (FTLD-tau; $n=2$; mean age $=88.5$ years) but not in control tissue (control; $n=2$; mean age $=73$ years). B) Similar aggregates are also detected in the cortical tissue of rTg4510 (4 months), ALZ17 (12 months), and pR5 (6 months) tau transgenic mice but not in tau knockout mice (tau KO; 6 months) or wild-type (WT) animals $(n=3)$. C) Comparison of RN235 (green) labeling to that of the p-Thr231 antibody, AT180, and the p-Ser202/205 antibody, AT8 (both red), in cortical tissue of a 3-month-old K3 mouse, revealing that RN235 labeling is consistent with that of AT8 and detects NFTs without a definable nucleus, whereas AT180 labeling is frequently more diffuse (arrowhead) and the nucleus is clearly observed, consistent with pre-NFTs. Nuclear staining is shown with DAPI (blue). Scale bars $=25 \mu \mathrm{m}$. 
A
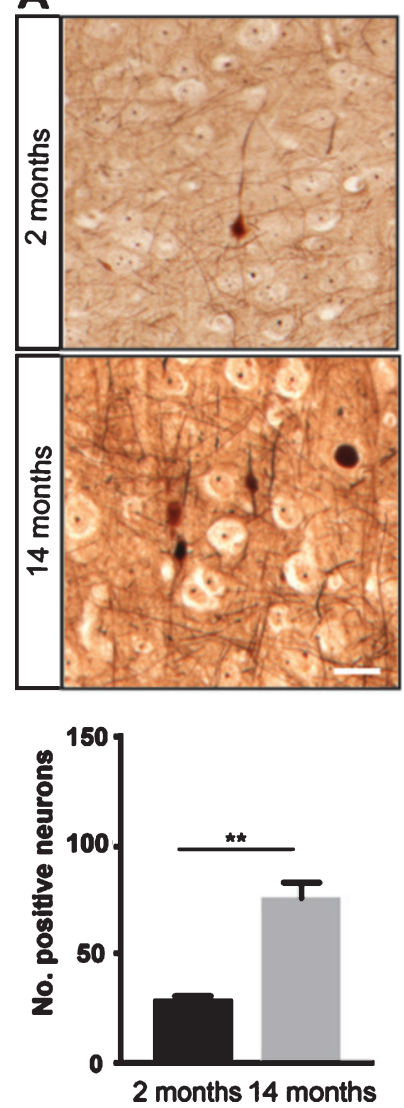

B
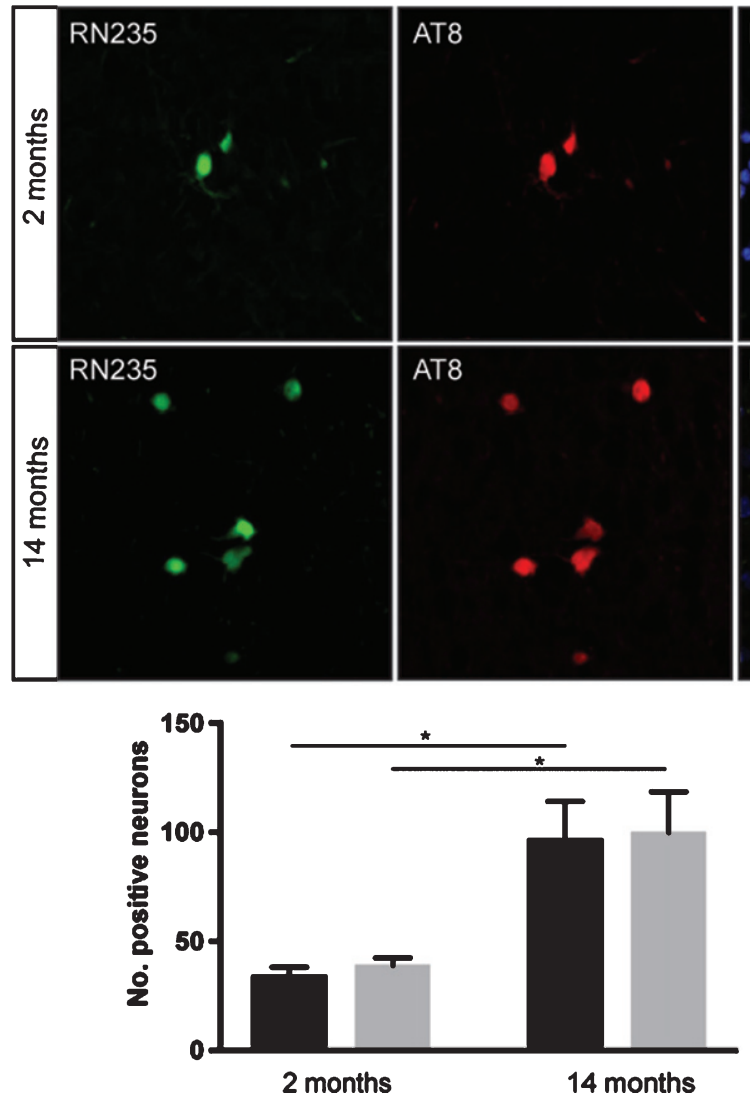
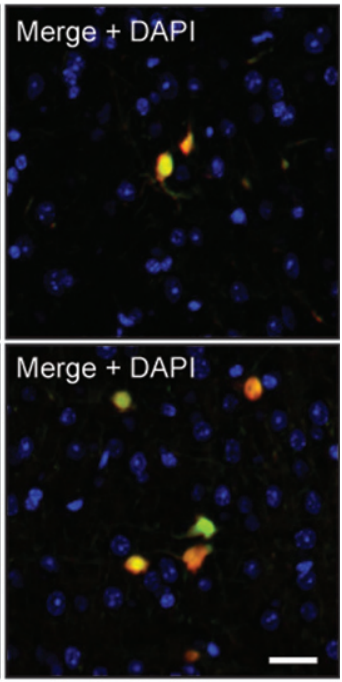

RN235

AT8

Fig. 3. RN235 detects a progressive tau pathology. A) Between 2 and 14 months, K3 mice exhibit a 2-fold increase in Bielschowsky's silver-stained NFTs in the cortex ( $n=3$; mean + SEM; ${ }^{* *} p<0.01$, Student's unpaired $t$-test). B) The number of RN235-positive NFTs also significantly increased from 2 to 14 months of age and is consistent with AT8 labeling $(n=3$; mean + SEM; * $p<0.05$ Two-way ANOVA with Tukey's multiple comparisons test). Scale bars $=25 \mu \mathrm{m}$.

primes the phosphorylation of Thr231 [11,12]. If this were the case, it would be expected that RN235 labeling would be similar to that of AT180, an antibody which detects $\mathrm{p}-\mathrm{Th} 231$. Instead, we have shown that RN235 labeling co-localizes with that of AT8 but rarely with AT180, suggesting that phosphorylation of Thr231 occurs early in disease, whereas p-Ser235 is associated with late stage p-tau changes consistent with the phosphorylation of Ser202/205. This is in agreement with a more recent study which demonstrated that GSK-3 $\beta$ acts on Thr231 in the absence of priming of Ser235 but is instead dependent on the phosphorylation at Thr175 [13]. Interestingly, an antibody specific to p-Thr175 has previously been shown to detect pre-NFTs, similar to AT180 [1].

Although immunotherapy targeting p-tau epitopes has proven to be successful in multiple pre-clinical studies, one would not expect the therapeutic potential of RN235 to be great, as the disease would be too far advanced for it to have an effect. Here we have shown, however, that the RN235 monoclonal antibody is ideal as a research tool for characterizing NFT formation in both human and mouse tissue.

\section{ACKNOWLEDGMENTS}

This research was supported by the estate of Dr Clem Jones AO, the State Government of Queensland, the Federal Government of Australia (ACT900116), the Australian Research Council (DP13300101932) and the National Health and Medical Research Council of Australia (APP1037746, APP1003150) to J.G. and The Yulgilbar Foundation to R.M.N. R.M.N. is a previous recipient of the Alzheimer's Australia Dementia Research Fellowship. We thank Tishila Palliyaguru, Linda Cumner, Adam Briner and Lorna Latemore for expert technical 
assistance, Trish Hitchcock and her team for animal care and Rowan Tweedale for critical reading of the manuscript. Imaging was performed at the Queensland Brain Institute's Advanced Microscopy Facility, generously supported by the Australian Government through an ARC LIEF grant (LE 100100074).

Authors' disclosures available online (https:// www.j-alz.com/manuscript-disclosures/17-0610r1).

\section{REFERENCES}

[1] Augustinack JC, Schneider A, Mandelkow EM, Hyman BT (2002) Specific tau phosphorylation sites correlate with severity of neuronal cytopathology in Alzheimer's disease. Acta Neuropathol 103, 26-35.

[2] Serrano-Pozo A, Frosch MP, Masliah E, Hyman BT (2011) Neuropathological alterations in Alzheimer disease. Cold Spring Harb Perspect Med 1, a006189.

[3] Hanger DP, Anderton BH, Noble W (2009) Tau phosphorylation: The therapeutic challenge for neurodegenerative disease. Trends Mol Med 15, 112-119.

[4] Amniai L, Barbier P, Sillen A, Wieruszeski JM, Peyrot V, Lippens G, Landrieu I (2009) Alzheimer disease specific phosphoepitopes of Tau interfere with assembly of tubulin but not binding to microtubules. FASEB $J \mathbf{2 3}$, 1146-1152.

[5] Sibille N, Huvent I, Fauquant C, Verdegem D, Amniai L, Leroy A, Wieruszeski JM, Lippens G, Landrieu I (2012) Structural characterization by nuclear magnetic resonance of the impact of phosphorylation in the proline-rich region of the disordered Tau protein. Proteins 80, 454-462.
[6] Lyons AJ, Gandhi NS, Mancera RL (2014) Molecular dynamics simulation of the phosphorylation-induced conformational changes of a tau peptide fragment. Proteins $\mathbf{8 2}$, 1907-1923.

[7] Liu C, Song X, Nisbet R, Götz J (2016) Coimmunoprecipitation with tau isoform-specific antibodies reveals distinct protein interactions and highlights a putative role for 2N tau in disease. J Biol Chem 291, 8173-8188.

[8] Nisbet RM, Van der Jeugd A, Leinenga G, Evans HT, Janowicz PW, Götz J (2017) Combined effects of scanning ultrasound and a tau-specific single chain antibody in a tau transgenic mouse model. Brain 140, 1220-1230.

[9] Ittner LM, Fath T, Ke YD, Bi M, van Eersel J, Li KM, Gunning P, Götz J (2008) Parkinsonism and impaired axonal transport in a mouse model of frontotemporal dementia. Proc Natl Acad Sci U S A 105, 15997-16002.

[10] Baker S, Polanco JC, Götz J (2016) Extracellular vesicles containing P301L mutant tau accelerate pathological tau phosphorylation and oligomer formation but do not seed mature neurofibrillary tangles in ALZ17 mice. J Alzheimers Dis 54, 1207-1217.

[11] Cho JH, Johnson GV (2004) Primed phosphorylation of tau at Thr 231 by glycogen synthase kinase $3 \beta$ (GSK $3 \beta$ ) plays a critical role in regulating tau's ability to bind and stabilize microtubules. J Neurochem 88, 349-358.

[12] Li T, Hawkes C, Qureshi HY, Kar S, Paudel HK (2006) Cyclin-dependent protein kinase 5 primes microtubuleassociated protein tau site-specifically for glycogen synthase kinase $3 \beta$. Biochemistry 45, 3134-3145.

[13] Moszczynski AJ, Gohar M, Volkening K, Leystra-Lantz C, Strong W, Strong MJ (2015) Thr175-phosphorylated tau induces pathologic fibril formation via GSK3 $\beta$-mediated phosphorylation of Thr231 in vitro. Neurobiol Aging 36, 1590-1599. 